\title{
ТЕЧЕНИЕ КОРОНАВИРУСНОЙ ИНФЕКЦИИ У ПАЦИЕНТКИ С САХАРНЫМ ДИАБЕТОМ 1 ТИПА НА ПОМПОВОЙ ИНСУЛИНОТЕРАПИИ
}

\author{
Гауэрт В.Р., Корсакова Д.А. \\ ФГБОУ ВО ЯГМУ Минздрава России, Ярославль
}

Всемирной организацией здравоохранения новая коронавирусная инфекция COVID-19 признана пандемией, которая достаточно часто приводит к развитию осложнений со стороны сердечно-сосудистой, дыхательной, эндокринной систем, системы гемостаза и других. Ученые и клиницисты находятся в постоянном поиске ответов на возникающие вопросы, тем не менее количество осложнений у пациентов, перенесших инфекцию растет.

В частности, инфекция COVID-19 ассоциирована с манифестацией различных нарушений углеводного обмена вплоть до развития сахарного диабета (СД). Кроме того, у пациентов, имеющих СД, как 1, так и 2 типа, новая коронавирусная инфекция может значительно ухудшать целевые показатели гликемии, что требует коррекции сахароснижающей терапии, в том числе инсулинотерапии.

В данной работе представлен клинический случай течения COVID-19 у пациентки с сахарным диабетом 1 типа на помповой инсулинотерапии.

ЦЕЛЬ: подробно изучить течение новой коронавирусной инфекции на фоне СД 1 типа, проанализировать влияние COVID-19 на гликемический профиль на конкретном клиническом примере, рассмотреть необходимость и особенности коррекции инсулинотерапии с учетом развивающейся инсулинорезистентности.

МАТЕРИАЛЫ И МЕТОДЫ: проанализирована история болезни пациентки К., наблюдавшейся в Рыбинской городской поликлинике № 4 с диагнозом:

Основной: COVID-19, положительный результат теста на вирус. Легкое течение.

Фоновый: Сахарный диабет, 1 типа, целевой $\mathrm{HbA}_{1 c}<6,5 \%$. Ухудшение течения.

Осложнения фонового: Дистальная периферическая диабетическая сенсомоторная полинейропатия. Артифакия OU.

PЕЗУЛЬТАТЫ: в ходе изучения данного клинического случая были выявлены следующие особенности течения СД, 1 типа, на фоне возникшей инфекции COVID-19: за сутки до появления основных симптомов коронавирусной инфекции была впервые зафиксирована гипергликемия до 15 ммоль/л, для ее купирования потребовалось увеличение дозы базального инсулина до 150\%, несмотря на легкое течение COVID-19 и отсутствие приема глюкокортикоидов. Болюсный же коэффициент был увеличен до 3:1, что, со слов пациентки, произошло впервые с момента использования помпы. Вернуться на прежние дозы инсулина пациентке не удается на протяжении трех месяцев после перенесенной инфекции COVID-19, что вероятнее всего, на наш взгляд, связано с развившейся у нее на фоне данной инфекции инсулинорезистентностью.

Выводы: повышение гликемии у пациентки с СД, 1 типа, до появления основных симптомов заболевания COVID-19, можно объяснить действием контринсулярных гормонов, быстрым развитием инсулинорезистентности на фоне острого инфекционного процесса, что подтверждается возросшей потребностью в инсулине для коррекции гликемии, невозможностью возвращения к дозировкам инсулина до COVID-19, и клинически приводит к развитию вариабельности гликемического профиля в течение суток. Резкое повышение гликемии на фоне инфицирования COVID-19 может трактоваться как один из ранних симптомов заболевания для пациента с СД, 1 типа, а развившаяся резистентность к инсулину, как возможное постковидное осложнение. 